\title{
Provision of combined antiretroviral therapy in HIV-positive pregnant women and the increased risk of apoptosis-related intra-uterine growth restriction
}

\author{
Artana Putra IW ${ }^{1}$, Ketut Suwiyoga ${ }^{2}$ \\ ${ }^{1}$ Maternal-Fetal Medicine Division, Department of Obstetrics and Gynecology, Udayana University/Sanglah General Hospital \\ Denpasar, Bali, Indonesia \\ ${ }^{2}$ Gynaecology-Oncology Division, Department of Obstetrics and Gynecology, Udayana University/Sanglah General Hospital \\ Denpasar, Bali, Indonesia
}

\begin{abstract}
Human immunodeficiency virus (HIV) infection during pregnancy is still a major problem worldwide, especially in developing countries. Although administration of anti-retroviral drugs has succeeded in reducing mother-to-child transmission, poor obstetric outcomes such as intrauterine growth restriction (IUGR) and preterm labor still significantly affect this population. IUGR and preterm labor are associated with many short-term and long-term adverse outcomes such as low APGAR score, longer neonatal intensive care unit (NICU) stay, and increased neonatal morbidity and mortality. These conditions are thought to be related to pathologic apoptosis in the placenta, induced either by the presence of viral antigens or by the effect of combined antiretroviral therapy (ART). Some viral antigens are thought to be involved in apoptosis by mediating bystander apoptosis and increasing reactive oxygen species (ROS) production. These viral antigens such as the glycoprotein Env and gp120 and gp41 subunits may trigger caspase-dependent and caspase-independent apoptotic pathways. In some studies, combined ART has been reported to exert mitochondrial DNA toxicity, causing DNA dysfunction. The toxicity of antiretroviral therapy was first described in 1988 through observations of damaged muscle fibers which are characteristic of myopathy, in patients who consume zidovudine. Thus, it is suggested that both viral antigens and combined ART may activate apoptosis cascades, ultimately disturbing placental functions.
\end{abstract}

HIV AIDS Rev 2019; 18, 1: 1-6 DOI: https://doi.org/10.5114/hivar.2019.84203

Key words: growth restriction, preterm, pregnancy, HIV, anti-retroviral, apoptosis.

\section{Introduction}

Human immunodeficiency virus (HIV) infection during pregnancy is still a major problem worldwide, especially in developing countries. Since its introduction in 1994, combined anti-retroviral therapy (cART) has been successful in decreasing viral load and reducing vertical transmission

dramatically from $14-48 \%$ to $0.4 \%$. Despite this significant reduction of vertical transmission, the incidence of intrauterine growth restriction (IUGR) and preterm birth in this population remains high. IUGR is closely related to placental pathology, such as inflammation, oxidative stress, thrombus formation, and apoptosis. These pathologic processes are

Article history:

Received: 20.10.2018

Received in revised form: 26.12.2018

Accepted: 27.12.2018

Available online: 10.03.2019
International Journal of HIV-Related Problems

HIV \& AIDS

R e vi e w 
usually related to various risk factors, such as maternal diabetes, hypertension, preeclampsia, and also HIV and its therapy. Some studies showed high incidence of IUGR and preterm birth in HIV-positive women who consume cART, although their relations with other factors have not been confirmed yet. The lack of data and knowledge regarding the safety of long-term cART provision has now raised concerns about the potential side effects for mother and neonate [1].

The incidence of IUGR in HIV-positive pregnant women varies. Geetha et al. reported low birth weight in 52.3\% of cases and IUGR in 20\% of cases [2]. Lopez et al. reported $23.4 \%$ for cases of IUGR diagnosed antenatally. Two thirds of those cases were associated with abnormal umbilical artery Doppler study, which indicates placental insufficiency [3]. The Women and Infants Transmission Study (WITS) reported $21 \%$ for cases of preterm births and $19 \%$ for cases of low birth weight, while Lambert et al. reported $17 \%$ and $13 \%$ for cases of low birth weight and IUGR in HIV-positive women respectively. Neonates with IUGR were associated with iatrogenic preterm birth and needed a longer NICU stay [3-5]. Mittal et al. reported that in a group of patients who received longer cART, the incidence of IUGR was $27 \%$, which was associated with apoptosis-related placental insufficiency [6].

IUGR is associated with adverse short-term or longterm outcomes in neonates. Short-term complications include preterm birth, low APGAR score, longer NICU stay, and increased neonatal morbidity and mortality. Long-term complications include increased incidence of metabolic and cardiovascular diseases in children [7]. In this paper, we aim to review current knowledge and evidence regarding the role of HIV infection and CART provision in the increasing incidence of adverse obstetrics outcomes in HIV-positive pregnant women.

\section{Placental pathology in intrauterine growth restriction}

The placenta is a vital organ that maintains optimal fetal growth during pregnancy. It serves as respiratory and excretory organ, and also facilitates glucose, amino acid and fatty acid transfer. It produces hormones vital for fetal growth, such as progesterone, growth hormone, and human placental lactogen. Thus, whenever an adverse obstetrical outcome occurs, placental function should be the first to be investigated, either prenatally by ultrasound or postnatally through histopathology examination. Many adverse obstetrical outcomes such as miscarriage, IUGR, preterm birth, and preeclampsia are closely related to pathologic processes in the placenta $[8,9]$.

Placentas from neonates with IUGR are usually smaller, hypoxic, have disturbed trophoblastic invasion, increased vascular resistance, reduced artery number, luminal diameter and branching, decreased villous quality, and increased trophoblast apoptosis [10]. Various mechanisms have been proposed to explain placental dysfunction, including abnormal early growth, maternal inflammation, ischemia, hemorrhage and thrombosis of varying degrees in the placenta.
In patients with severe IUGR and preeclampsia, extra-villous trophoblasts (EVT) exhibit lower stromal invasion resulting from immunity-induced apoptosis by maternal macrophages. The lack of EVT invasion will be followed by a fibrous deposit on the wall of the spiral artery, accumulation of foam cells and muscularization of distal segment of the spiral artery, all of which cause decidual vasculopathy. Prolonged hypoxia in the intervillous space may also cause oxidative stress and placental apoptosis in this setting [11].

In these patients, the abnormal villous differentiation is distinct with placental changes secondary to abnormal uteroplacental perfusion. Figures obtained from electron microscope examination on villous tissue and blood vessel show arrested angiogenesis that inhibits villous branching. This pathology is described as distal villous hypoplasia, and is usually associated with severe cytotrophoblast reduction and syncytial knotting. The resultant effect of this unstable perfusion is ischemia-reperfusion injury that results in cytotrophoblast cell cycle arrest, syncytial fusion problem, and endoplasmic reticulum stress. The combination of these conditions will accelerate apoptosis in the placenta [11]. Studies on animals and humans indicate that placental restriction and insufficiency may be caused by a series of placental changes such as placental growth and substrate transport disturbance, increased apoptosis and autophagy, and also increased glucocorticoid activity [12].

Apoptosis of trophoblastic villous is significantly increased in pregnancies complicated by preeclampsia and IUGR. They show abnormal capillary vessels and atrophic villi, disturbing oxygen supply to the placenta and triggering apoptosis [13]. The apoptotic index is also increased in the placenta [14]. When normal trophoblasts are cultured in a hypoxic environment, they tend to show increased regulatory activity of $\mathrm{p} 53$ and BCL-2-like protein (BAX) expression, and also anti-apoptotic BCL-2 expression, which promote apoptosis. P53 expression and the active form of caspase-3 are dysregulated within villous trophoblasts [12].

Another study by Crocker et al. concluded that apoptosis was significantly increased in both preeclampsia and IUGR. This trophoblastic apoptosis may reflect poor placental condition. A similar result was also reported by Smith et al. They explain that increased apoptosis in these patients was a protective phenomenon to allow better nutrient transport across the placental barrier, resulting in smaller placentas and neonates. Apoptotic index values of $0.17 \%, 0.24 \%$ and $0.39 \%$ were reported in normal pregnancies, IUGR, and preeclampsia, respectively. The initiation phase of the apoptotic cascade occurs in cytotrophoblasts, whereas the execution phase occurs in syncytiotrophoblasts. Apoptotic nuclei produced are then accumulated as a syncytial knot [13].

\section{Review of apoptosis}

Apoptosis was first introduced by the German scientist Carl Vogt in 1842. Unlike necrosis, apoptosis is a genetically coded and an evolutionary mechanism of cell death. Apoptosis is marked by various morphological and biochemi- 
cal aspects: cellular shrinkage, DNA fragmentation, nuclear and cytoplasmic condensation, cytosolic calcium increase, caspase activation, apoptotic body formation, and phagocytosis by macrophages. Apoptosis is induced by a multiple-signal pathway and is regulated by complex intrinsic and extrinsic ligands. Apoptosis may occur through intrinsic (mitochondrial) or extrinsic (death receptor-mediated) pathways. The intrinsic pathway is activated by various endogenous and exogenous stimuli, including DNA damage, ischemia, and oxidative stress, whereas the extrinsic pathway is initiated by death receptor activation, binding of extracellular death signals such as TNF- $\alpha$, Fas ligand (Fas-L), TNF-related apoptosis-inducing ligand (TRAIL) and Apo-3 ligand (Apo-3L) with their receptors $[15,16]$.

According to caspase involvement, there are two main apoptosis pathways: caspase-dependent apoptosis and caspase-independent apoptosis. Caspase-dependent apoptosis is the classic pathway of programmed cell death, usually involving cysteine-dependent aspartate-specific protease (caspase)-8, caspase- 9 , caspase-12, caspase- 7 and caspase- 3 . There are various receptors that participate in this apoptotic pathway such as TNF- $\alpha$ receptors, FasL receptors, toll-like receptors (TLR), death receptors and others. Proapoptosis caspase-3 is an executioner caspase which is mainly activated during apoptosis, triggered by mediator receptors of the extrinsic pathway and the mitochondrial intrinsic pathway. Meanwhile, B-cell lymphoma-2 (Bcl-2) is an anti-apoptotic protein that prevents apoptosis through caspase inhibition and maintenance of mitochondrial membrane integrity $[14,17,18]$.

TNF- $\alpha$ induces a caspase-8-dependent pathway dependent on TNF- $\alpha$ receptors and activates caspase- 8 through a death complex and $\mathrm{Bcl}-2$ protein. Activation of the $\mathrm{Bcl}-2$ protein family can cause the mitochondrial membrane to change and stimulate cytochrome $\mathrm{C}$ release. Cytochrome $\mathrm{C}$ is a proapoptotic signal which can activate caspase cascade reactions, inducing apoptosis. Some ultraviolet or X-ray radiation may depolarize mitochondria and affect membrane permeability, increasing reactive oxygen species (ROS). This will trigger the release of cytochrome $\mathrm{C}$ through caspase- 9 and caspase- 3 activation $[15,17]$. Similarly, some pathogens may also induce apoptosis via the caspase- 8 dependent pathway due to RNA and DNA damage. The RNA fragment produced by Mycobacterium tuberculosis has the capability to trigger caspase-8-dependent apoptosis. In vivo, DNA damage triggers apoptosis by increasing ROS levels and changing the permeability of the mitochondrial membrane [15].

In caspase-independent apoptosis, caspase is not involved in the process of apoptosis. There are many intracellular ligands that may trigger changes in the mitochondrial membrane. Apoptosis-inducing factor (AIF) has been found to be the main proapoptosis factor which is released by $\mathrm{mi}-$ tochondria. Its translocation to the cellular nucleus causes irreversible DNA damage and apoptosis occurs. ROS may also act as a trigger for caspase-independent apoptosis; ROS mediates the activation of poly-ADP-ribose polymerase-1
(PARP-1). PARP-1 activation is very important to facilitate AIF release from mitochondria. Mitochondrial damage is the first phase of apoptosis, followed by an increase in ROS production. ROS play a role in both cascade-dependent and cascade-independent apoptosis. ROS also act as a bridge that connects the two types of apoptosis in vivo. They are mainly produced in mitochondria, emphasizing the role of mitochondria in the crosstalk of both apoptotic pathways [15].

\section{Antiretroviral-induced mitochondrial dysfunction}

Mitochondria are cellular organelles located in the cytoplasm of most eukaryocyte cells. Mitochondria maintain cellular viability through various important processes such as heat production, energy supply, cell respiration, calcium homeostasis and also anabolism and catabolism of various metabolite products. They provide energy through the formation of the adenosine triphosphate (ATP) molecule, which is the largest energy source of a cell. In pathological conditions, mitochondria are also a major source of ROS production and capable of inducing apoptosis $[19,20]$.

The toxicity of antiretroviral therapy was first described in 1988 through observations of damaged muscle fibers which are characteristic of myopathy, in patients who consume zidovudine. The myopathy was thought to be closely related to mitochondrial toxicity. This was supported by some findings in muscle biopsies of patients receiving ART therapy, such as mitochondrial defect, decreased oxidative phosphorylation (OXPHOS) activity and decreased mitochondrial DNA (mtDNA) level [21, 22]. Early studies on non-nucleotide reverse transcriptase inhibitors (NRTI) drugs have shown that they exert very minimal effects on DNA polymerase alpha, but affect beta and gamma polymerase to several degrees. DNA polymerase alpha is the main enzyme for DNA replication, while polymerase beta has an important role in DNA repair. The clinical impact of DNA polymerase beta inhibition is still unknown. The effect of the NRTIs on DNA polymerase gamma has been investigated and it is suspected that the resistance to this enzyme will trigger mitochondrial DNA depletion. If this mitochondrial DNA depletion drops beyond the critical limit, it will lead to energy insufficiency and cell dysfunction. Animal studies have revealed some degree of mitochondrial toxicity, as evidenced by a decrease in mtDNA and abnormalities of the OXPHOS enzyme in the placenta of monkeys exposed to zidovudine [23].

Another theory proposes that the binding and inhibition of polymerase- $\gamma$ enzymes by NRTIs is not linearly correlated with clinical manifestations of mitochondrial toxicity. Mitochondrial toxicity caused by NRTIs does not always follow the chronological stages of the polymerase theory. Not all cases of decreased mtDNA levels trigger changes in the expression or activity of the proteins vital for mitochondrial respiration. However, mitochondrial transcription gene disorders and impairment of the respiration chain activity have been observed despite the absence of mtDNA depletion or 
polypeptide synthesis decrease. In this condition, NRTIs disturb mitochondrial function via other mechanisms such as nucleoside homeostasis disturbance caused by the pharmacokinetics of NRTIs. NRTIs can also directly inhibit mitochondrial enzymes, which may increase the generation of ROS [22-25].

In vitro studies have shown that inhibition of polymerase- $\gamma$ will decrease the amount of mtDNA, which subsequently causes DNA dysfunction. MtDNA encodes the subunit of the respiratory reaction, and if defected will disturb oxidative phosphorylation (OXPHOS). Evidence has shown that mitochondrial function is affected by HIV particles, cytokines and/or cART toxicity [23]. Mitochondrial dysfunction can also be caused by the indirect effects of NRTIs, through competition with endogenous deoxyribonucleotide triphosphates (dNTPs) independent of polymerase- $\gamma$ inhibition. Therefore, NRTIinduced mitochondrial dysfunction cannot only be explained through the polymerase- $\gamma$ hypothesis [26]. NRTIs had different intensity and capacity to cause mitochondrial toxicity. The toxicity sequence from the strongest to the weakest is as follows: $2^{\prime}-3^{\prime}$ dideoxycytidine (ddC), didanosine (ddI), stavudine (d4T), zidovudine (AZT), lamivudine ( $3 \mathrm{TC}$ ), and abacavir and tenofovir (TDF) had the same strength $[19,23,27]$.

Placental formation is genetically controlled. There is a significant increase in mitochondrial mass along with increasing gestational age. The placenta grows at a high speed to accommodate the increased fetal nutritional and oxygen demand. Some studies have suggested a causal relationship between abnormal placental mitochondria and impaired placental function, as in fetal growth restriction and preeclampsia cases. In vitro studies have reported that placenta exposed to zidovudine shows accelerated ROS production, impaired mitochondrial functions and caspase-dependent cell death. These events will cause ultrastructural degenerative changes of the placental villi in HIV-positive pregnant women treated with zidovudine [1,27].

Sandra Hernandez et al. found that there was a decrease in mtDNA by as much as $39.20 \% \pm 2.78 \%$ and caspase- 3 dependent apoptosis by as much as $0.48 \% \pm 0.10 .23$ Tamuzi and Tshimwanga reported five observational studies and all those studies showed that ART increased the defect in mitochondria, which was statistically significant $(p<0.05)$ [27]. This mitochondrial toxicity can also be evaluated using the mtDNA/nDNA (nuclear DNA) ratio. Pregnant women with HIV infection had a lower mtDNA/nDNA ratio compared with pregnant women without HIV infection. It was also found that the mtDNA/nDNA ratio was lower in pregnant women who started antiretroviral therapy before pregnancy compared with those who started antiretroviral therapy after pregnancy [1]. Many studies have supported the role of NRTI-induced mitochondrial toxicity in this population. But, because of the low number of sample in these studies, it is still difficult to completely describe their relationship with poor pregnancy outcomes [19].

\section{HIV antigen and apoptosis}

The role of viremia in apoptosis of CD4 cells in HIV virus infection is supported by the observation of increased apoptosis in patients with untreated viral infections. The number of CD4 cells infected with HIV is relatively low compared to the number of CD4 cells that undergo apoptosis. This raises a belief that the CD4 decrease during HIV infection is a result of bystander apoptosis. A study by Finkel et al. showed that most of the cells that experienced apoptosis during HIV infection were not infected, but they were located adjacent to the infected cells. Therefore bystander apoptosis is believed to be the most important mechanism of CD4 decrease in acquired immunodeficiency syndrome (AIDS). Some viral antigens involved in this process are Env glycoprotein and gp120 and gp41 subunits [28, 29]. Gp120 subunit from the HIV Env is attached to CD4 and its co-receptor, which behaves as a potential candidate to mediate bystander apoptosis. Inhibition of gp120 attachment to CD4 or its co-receptor could prevent HIV-induced apoptosis [28, 29].

Current evidence shows that HIV-1 Env mediates apoptosis of bystander cells through increased mitochondrial ROS production, increased global cellular metabolism, and increased mitochondrial fusion. Env-mediated ROS production can be inhibited by antagonizing CXCR4 and AMD3100 . However, it is unclear whether the increased ROS production is the result of the Env signal that binds CXCR4 or the oxidative stress that is induced by HIV-1 Env. HIV Env is a known upregulation signal for stress protein families such as heat shock protein 70 (Hsp70) [29].

Even though the role of tumor necrosis factor receptor 1 (TNFR1) and the Fas (CD95) pathway have not been elucidated in Env-mediated apoptosis, there exists proof that HIV-infected cells show greater susceptibility towards Fas-induced apoptosis. Fas are upregulated in both the cellular membrane and the dissolved form in HIV-positive patient. Macrophages are believed to have a crucial role in cellular death in this pathway. TNF expression in the activated macrophage surface can induce apoptosis in bystander T-cells through TRAIL (TNF-related apoptosis-inducing ligand) DR5 (death receptor) activation. A further decrease in cell viability has been observed, induced by other viral proteins such as Nef (negative regulatory factor) and viral protein $\mathrm{R}$ (Vpr) that mimics biological effects of TNF, while gp120 and viral protein $\mathrm{U}(\mathrm{Vpu})$ could aggravate the pro-apoptotic effect of TNF. It has been shown that cross relation of CD4 through Gp120 may activate the CD95 (cluster of differentiation) pathways/CD95L (Fas ligand) and Nef expressing $\mathrm{T}$ cells, which results in the upregulation of CD95L, ultimately causing apoptosis [29].

Many studies have suggested that cellular and mitochondrial functions are affected by both HIV and highly active antiretroviral therapy (HAART), leading to adverse clinical events. However, few data are available about mitochondrial dynamics in the context of infectious diseases. To date, only in vitro models demonstrating $\mathrm{HIV}-1$ viral protein $\mathrm{R}(\mathrm{Vpr})$ interaction with apoptosis and mitochondrial dynamics 
have been reported. No ex vivo studies have been performed to assess the influence of HIV on mitochondrial dynamics or the relationship between HAART and mitochondrial fusion and fission events and their potential association with adverse obstetric outcomes in HIV-infected pregnant women. In a cohort of HIV-infected pregnant women with cART, there was a significant decrease in levels of mitofusin-2 (Mfn2) in maternal peripheral blood mononuclear cells in the first trimester of pregnancy, which continued to decline throughout pregnancy. Likewise, there was a significant decrease in the concentration of Mfn2 and dynamin-related protein 1 (Drp1) in the placenta of HIV-infected pregnancies on cART. As previously described in vitro, Vpr can interfere with mitochondrial dynamics in vivo, but only in tissues in contact with the virus. Experimental data show that decreased expression of Mfn2 accelerates apoptosis and inhibits cellular development by blocking mitochondrial metabolism and disrupting mitochondrial membrane potential and cellular activity, disrupting embryonic development. The effects of an unbalanced fusion and fission process in this population may explain the occurrence of poor obstetric outcomes [20].

Interestingly, it was found that in HIV-positive pregnant women who received cART throughout pregnancy, the level of viral proteins, including $\mathrm{Vpr}$, were almost undetectable. Thus, the imbalance of mitochondrial dynamics and increased apoptosis that is observed in these women may be due to cART itself rather than being induced by viral antigens. Nevertheless, Vpr or other viral proteins may exert toxic effects even in small amounts [20].

The role of the virus in triggering apoptosis is also mediated by increased ROS production. ROS contains highly reactive oxygen molecules such as hydrogen peroxide $\left(\mathrm{H}_{2} \mathrm{O}_{2}\right)$, superoxide $\left(\mathrm{O}^{2-}\right)$ anion, nitric oxide $(\mathrm{NO})$ and hydroxyl radical $(\mathrm{OH})$. Excessive ROS production causes oxidative stress, which is commonly found during infectious inflammation. Oxidative stress damages cellular structures, including lipids, proteins and DNA [30-32]. Mitochondria are the major source of ROS during oxidative phosphorylation. In the state of pathology, mitochondria not only act as the origin of overproduced ROS but also become the target of oxidative stress, leading to mitochondrial swelling, superoxide outflow and cytochrome leakage, modifying protein structures and initiating the apoptotic cascade $[33,34]$.

Neutrophils from HIV-1 infected patients also appear to be activated in vivo, showing increased cellular surface expression of programmed death ligand 1 (PD-L1), adhesion molecules CD11b and CD18, low CD62L expression, and spontaneous production of TNF- $\alpha$. An exacerbated susceptibility to apoptosis in the absence of stimuli has also been reported, and it is interesting to note that the phagocytosis of apoptotic neutrophils by macrophages is an additional source of inflammation [35].

In several reports, ROS worsens inflammatory status by increasing the production of pro-inflammatory cytokines including IL-1 $\beta$, IL- 6 , interferon (IFN) and TNF- $\alpha$, in turn inducing further ROS formation and exaggerated inflammatory and apoptotic processes $[31,36]$.

\section{Conclusions}

IUGR and preterm labor are still an issue in HIV-positive pregnant women. This poor obstetric outcome is related to pathological apoptotic processes in the placenta. It may be triggered directly by the viral particle or, as more recently proposed, as the result of cART provision. Some viral antigens such as glycoprotein Env and gp120 and gp41 subunits may trigger bystander apoptosis. This may also be mediated by the increase of ROS production. Excessive ROS production causes oxidative stress, damaging cellular structure, lipids, membrane proteins and DNA, and in turn activating caspase-dependent and caspase-independent cascades. The administration of cART, especially NRTIs, has been shown to exert some degree of toxicity to mitochondrial DNA. Mitochondrial damage will induce apoptosis via the intrinsic pathway. Although the results from many studies are suggestive of this effect, some studies have reported opposite results. Thus, further research is needed to confirm the relationship between cART provision and some adverse obstetric outcomes. Nevertheless, clinicians should be aware of this potential situation when treating HIV-positive pregnant women.

\section{Conflict of interest}

The authors declare no potential conflicts of interest with respect to the research, authorship, and/or publication of this article.

\section{References}

1. Money DM, Wagner EC, Maan EJ, et al. Evidence of subclinical mtDNA alterations in HIV-infected pregnant women receiving combination antiretroviral therapy compared to HIV-negative pregnant women. PLoS One 2015; 10: e0135041.

2. Shivamurthy G, Pukale R, Mankhani R. A prospective study of obstetric and new born outcome in a cohort of HIV affected pregnant women. J Evolution Med Dent Sci 2015; 4: 514-524.

3. Lopez M. Risk of intra uterine growth restriction among HIVinfected pregnant woman: a cohort study. Eur J Clin Microbiol Infect Dis 2015; 34: 223-230.

4. Lambert JS, Watts DH, Mofenson L, et al. Risk factor for preterm birth, low birth weight, and intrauterine growth retardation in infants born to HIV-infected pregnant women receiving zidovudine. Pediatric AIDS Clinical Trials Group 185 Team. AIDS 2000; 14: 1389-1399.

5. Pooli R, Devi J, Rani S. Comparative study of pregnancy and its outcome between HIV positive and HIV negative pregnant woman. J Evolution Med Dent Sci 2016; 5: 717-721.

6. Mittal M, Mall AK, Sharma YG. Maternal and fetal outcome in HIV positive female. Int J Res Med Sci 2016; 4: 5237-5240.

7. Menendez-Castro C, Rascher W, Hartner H. Intrauteraine growth restriction - impact on cardiovascular disease later in life. Mol Cell Pediatr 2018; 5: 4.

8. Ackerman W 4th, Kwiek JJ. Role of the placenta in adverse perinatal outcome among HIV-1 seropositive women. J Nippon Med Sch 2013; 80: 90-94. 
9. Laxmichaya D, Sawant S, Venkat S. Study of placenta in intrauterine growth restricted pregnancy. J Basic Clin Reprod Sci 2017; 6: 69-76.

10. Monson T, Wright T, Galan HL, et al. Caspase dependent and independent mechanisms of apoptosis across gestation in a sheep model of placental insufficiency and intrauterine growth restriction. Apoptosis 2017; 22: 710-718.

11. Kingdom J, Walker M, Drewlo S, Keating S. Intra Uterine Growth Restriction: Placental Basis and Implications for Clinical Practice in Fetal Therapy. Cambridge University Press, Cambridge 2013; 347-349.

12. Zhang S, Regnault TR, Barker PL. Placental adaptations in growth restriction. Nutrients 2015; 7: 360-389.

13. Swati P, Hemalatha A, Kumar ML, Munikrishna M. Apoptosis in trophoblast in pre-eclampsia and intrauterine growth retardation: a light microscopy study. Ann Pathol Lab Med 2018, 5: A241-245.

14. El-Baz MA, El-Deeb TS, El-Noweihi AM, et al. Enviromental factors and apoptotic indices in patient with intrauterine growth retardation: a nested case-control study. Environ Toxicol Pharmacol 2015; 39: 589-596.

15. Hongmei Z. Extrinsic and Intrinsic Apoptosis Signal Pathway Review. InTechOpen 2012; 1-22.

16. Loreto C, Rocca GL, Anzalone R. The role of intrinsic pathway in apoptosis activation and progression in Pyeronie's disease. BioMed Res Int 2014; 2014: e1-10.

17. Rongvaux A, Jackson R, Harman CC, et al. Apoptotic caspases prevent the induction of type I interferons by mitochondrial DNA. Cell 2014; 159: 1563-1577.

18. White MJ, McArthur KM, Metcalf D, et al. Apoptotic caspases suppress mtDNA-induced STING-mediated type I IFN production. Cell 2014; 159: 1549-1562.

19. Morén C, Noguero-Julián A, Garrabou G, et al. Mitochondrial disturbances in HIV in pregnancies. AIDS 2015; 29: 5-12.

20. Mampel MG, Hernandez AS, Moren C, et al. Imbalance in mitochondrial dynamics and apoptosis in pregnancies among HIV-infected women on HAART with obstetric complications. J Antimicrob Chemother 2017; 72: 2578-2586.

21. Pickering A. Mitochondrial Oxidative Damage, Base-Excision Repair, and Antiretroviral Therapy. ProQuest LLC, 2016.

22. Smith RL, Tan JM, Jonker MJ, et al. Beyond the polymerase- $\gamma$ theory: Production of ROS as a mode of NRTI-induced mitochondrial toxicity. PLoS One 2017; 12: e0187424.

23. Hernandez S, Garcia MC, Moren C, et al. Placental mitochondrial toxicity, oxidative stress, apoptosis, and adverse perinatal outcomes in HIV pregnancies under antiretroviral treatment containing zidovudine. J Acquir Immune Defic Syndr 2017; 75: e113-e119.

24. Koczor CA, Jiao Z, Fields E, et al. AZT-induced mitochondrial toxicity: an epigenetic paradigm for dysregulation of gene expression through mitochondrial oxidative stress. Physiol Genomics 2015; 47: 447-454.

25. Rodríguez-Mora S, Mateos E, Moran M, et al. Intracellular expression of Tat alters mitochondrial functions in $\mathrm{T}$ cells: a potential mechanism to understand mitochondrial damage during HIV-1 replication. Retrovirology 2015; 12: 78.

26. Selvaraj S, Ghebremichael M, Li M, et al. Antiretroviral therapy induced mitochondrial toxicity: potential mechanisms beyond polymerase- $\gamma$ inhibition. Clin Pharmacol Ther 2014; 96: 110-120.

27. Morén C, Noguera-Julián A, Garrabou G, et al. Mitochondrial disturbances in HIV pregnancies. AIDS 2015; 29: 5-12.

28. Tamuzi JL, Tshimwanga JL. Antiretroviral therapy for mitochondrial toxicity in HIV-infected pregnant women. J Mol Genet Med 2017; 11: 315 .

29. Garg H, Mohl J, Joshi A. HIV-1 induced bystander apoptosis. Viruses 2012; 4: 3020-3043

30. Garg H, Joshi A. Host and viral factors in HIV-mediated bystander. Apoptosis 2017; 9: pii: E237.

31. Mampel MG, Hernandez AS, Moren C, et al. Imbalance in mitochondrial dynamics and apoptosis in pregnancies among HIV- infected woman on HART with obstetric complications. J Antimicrob Chemother 2017; 72: 2578-2586.

32. Rajopadhye S, Mukherjee S, Dankekar S, et al. Oxidative stress in HIV/AIDS patients in Mumbai. Juniper Online Journal of Immunovirology 2015; 1: 1-6.

33. Dagenais-Luussier X, Mouna A, Routy JP, et al. Current topicsin HIV-1 pathogenesis: the emergence of deregulated immunometabolism in HIV-infected subjects. Cytokine Growth Factor Rev 2015; 26: 603-613.

34. Ivanov AV, Elliston VT, Ivanova ON, et al. Oxidative stress during HIV infection: mechanisms and consequences. Oxid Med Cell Longev 2016; 2016: 8910396.

35. Couret J, Chang TL. Reactive oxygen species in HIV infection. EC Microbiol 2016; 3: 597-604.

36. Wu F, Tian FJ, Lin Y, et al. Oxidative stress: placenta function and dysfunction. Am J Reprod Immunol 2015; 76: 258-271. 